\title{
Ivacaftor in Subjects With Cystic Fibrosis Who Are Homozygous for the F508del-CFTR Mutation
}

\author{
Patrick A. Flume, MD, FCCP; Theodore G. Liou, MD, FCCP; Drucy S. Borowitz, MD; \\ Haihong Li, PhD; Karl Yen, MD; Claudia L. Ordoñez, MD; and David E. Geller, MD; \\ for the VX08-770-104 Study Group*
}

\begin{abstract}
Background: Ivacaftor (VX-770) is a cystic fibrosis transmembrane conductance regulator (CFTR) potentiator that was approved in the United States for the treatment of cystic fibrosis $(\mathrm{CF})$ in patients $\geq 6$ years of age who have a G551D mutation; however, the most prevalent diseasecausing CFTR mutation, F508del, causes a different functional defect. The objectives of this study were to evaluate the safety of ivacaftor in a larger population and for a longer time period than tested previously and to assess the efficacy of ivacaftor in subjects with CF who are homozygous for F508del-CFTR.

Methods: This was a phase 2 study with a 16-week randomized (4:1), double-blind, placebo-controlled period (part A) and an open-label extension (part B) for subjects who met prespecified criteria. Results: Part A: The safety profile of ivacaftor was comparable to that of the placebo. The overall adverse event frequency was similar in the ivacaftor $(87.5 \%)$ and placebo $(89.3 \%)$ groups through 16 weeks. The difference in the change of $\mathrm{FEV}_{1} \%$ predicted from baseline through week 16 (primary end point) between the ivacaftor and placebo groups was $1.7 \%(P=.15)$. Sweat chloride, a biomarker of CFTR activity, showed a small reduction in the ivacaftor vs placebo groups of $-2.9 \mathrm{mmol} / \mathrm{L}(P=.04)$ from baseline through week 16. Part $\mathrm{B}$ : No new safety signals were identified. The changes in $\mathbf{F E V}_{1}$ or sweat chloride in part $A$ were not sustained with ivacaftor treatment from week 16 to week 40.

Conclusions: These results expand the safety information for ivacaftor and support its continued evaluation. Lack of a clinical effect suggests that a CFTR potentiator alone is not an effective therapeutic approach for patients who have CF and are homozygous for F508del-CFTR.

Trial registry: ClinicalTrials.gov; No.: NCT00953706; URL: www.clinicaltrials.gov.
\end{abstract}

CHEST 2012; 142(3):718-724

Abbreviations: $\mathrm{ALT}=$ alanine aminotransferase; $\mathrm{AST}=$ aspartate aminotransferase $\mathrm{CF}=$ cystic fibrosis; $\mathrm{CFTR}=\mathrm{cystic}$ fibrosis transmembrane conductance regulator; HBE = human bronchial epithelial; ULN = upper limit of normal

$\mathbf{C}$ yystic fibrosis $(\mathrm{CF})$ is caused by mutations in the cystic fibrosis transmembrane conductance regulator (CFTR) gene. The encoded protein, CFTR, is an epithelial chloride channel that maintains hydration of lung secretions by regulating water absorption. ${ }^{1}$ Inadequate airway hydration results in obstruction of the airways with thick mucus, rendering the airways vulnerable to chronic infection and inflammation, leading to irreversible structural changes and respiratory failure.

CF-causing CFTR mutations cause either diminished or defective CFTR protein in the apical membrane, resulting in decreased chloride transport. ${ }^{2}$
The most prevalent disease-causing CFTR mutation is F508del (c.1521_1523delCTT). ${ }^{3-5}$ Approximately $90 \%$ of patients with $\mathrm{CF}$ in North America have F508del on at least one allele, and nearly one-half are homozygous. ${ }^{6}$ The F508del-CFTR mutation causes improper protein folding, resulting in little or no CFTR protein reaching the cell surface. ${ }^{7,8}$ Furthermore, any F508del-CFTR protein reaching the cell surface may also have a defect in the opening and closing of the channel, known as a gating defect. ${ }^{9}$

Compounds designed to increase CFTR channel opening, CFTR potentiators, can prevent the hyperabsorption of water across the epithelial surface. ${ }^{9}$ 
Ivacaftor is a CFTR potentiator shown to increase chloride transport by activated CFTR channels at the cell surface in vitro. ${ }^{10} \mathrm{G} 551 \mathrm{D}-\mathrm{CFTR}$ (c.1652G $>\mathrm{A}$ ) is the most common gating mutation associated with $\mathrm{CF}$ disease, having an allelic frequency of only approximately $2.2 \% .^{11}$ Ivacaftor was tested for up to 4 weeks in a small number of subjects with $\mathrm{CF}$ who had the G551D-CFTR mutation on at least one CFTR allele ${ }^{12}$ and has been approved in the United States for the treatment of $\mathrm{CF}$ in patients who are $\geq 6$ years of age and have a G551D mutation.

This study aimed to evaluate the safety profile of ivacaftor in a larger $\mathrm{CF}$ population (ie, subjects homozygous for the F508del-CFTR mutation). This patient population was chosen (1) because F508del-CFTR is the most common $\mathrm{CF}$-causing mutation, and patients with F508del on both alleles account for about 50\% of the population with $\mathrm{CF}$; (2) to explore potential efficacy because in vitro studies have suggested that a small amount of F508del-CFTR may reach the cell surface in some patients with the F508del mutation, and tissues derived from these patients show a limited response to CFTR potentiation ${ }^{9,13,14}$; and (3) because F508del-CFTR may respond to ivacaftor in some subjects, and most subjects enrolled in the G551D study had F508del on the other CFTR allele, a study of ivacaftor in subjects homozygous for the F508del-CFTR mutation may help isolate possible potentiation of F508del without a second mutation confounding the results.

\section{Materials AND METHODS}

\section{Study Design}

This was a two-part, phase 2, multicenter, randomized (4:1), placebo-controlled, double-blind study of ivacaftor (Kalydeco,

\section{Manuscript received October 19, 2011; revision accepted January 30,} 2012.

Affiliations: From the Departments of Medicine and Pediatrics (Dr Flume), Medical University of South Carolina, Charleston, SC; the University of Utah (Dr Liou), Salt Lake City, UT; the Department of Pediatrics (Dr Borowitz), State University of New York at Buffalo, Buffalo, NY; Vertex Pharmaceuticals Incorporated (Drs Li, Yen, and Ordoñez), Cambridge, MA; and the Divisions of Biomedical Research and Pediatric Pulmonology (Dr Geller), Nemours Children's Clinic, Orlando, FL.

*A complete list of study participants is located in e-Appendix 1 . Funding/Support: The DISCOVER study is sponsored by Vertex Pharmaceuticals Incorporated. This research was supported by the Cystic Fibrosis Foundation to the Women and Children's Hospital of Buffalo [Grant BOROWI03CSO] and to the Medical University of South Carolina [Grant C104-TDC10Y].

Correspondence to: Patrick A. Flume, MD, FCCP, Departments of Medicine and Pediatrics, Medical University of South Carolina, 96 Jonathan Lucas St, Room 812, Clinical Science Bldg, Charleston, SC 29425; e-mail: flumepa@musc.edu

(C) 2012 American College of Chest Physicians. Reproduction of this article is prohibited without written permission from the American College of Chest Physicians. See online for more details. DOI: $10.1378 /$ chest.11-2672 formerly known as VX-770; Vertex Pharmaceuticals Incorporated), $150 \mathrm{mg}$ po administered every $12 \mathrm{~h}$ for 16 weeks (part A), followed by an open-label 96 -week extension for subjects who met prespecified eligibility criteria (part B). The rationale for selecting subjects for the open-label extension was to determine whether the changes from baseline observed in part A of the study persisted. Prespecified eligibility criteria for enrollment in part B included either $\geq 10 \%$ change relative to baseline in $\mathrm{FEV}_{1} \%$ predicted at any time point through week 16 , or a sweat chloride concentration reduction from baseline $\geq 15 \mathrm{mmol} / \mathrm{L}$ at both the day 15 and week 8 visits. Interim analyses were planned to occur every 6 months, the first of which was performed at week 40 . The protocol was approved by each site's institutional review board, and subjects provided written informed consent and assent as applicable.

The study included clinically stable subjects aged $\geq 12$ years with CF, homozygous for the F508del-CFTR mutation, with an $\mathrm{FEV}_{1} \geq 40 \%$ predicted at screening. Subjects remained on their prestudy stable medication regimens throughout the study, including cycling inhaled antibiotics. Use of inhaled hypertonic saline or known inhibitors or inducers of cytochrome P450 3A4 was not allowed.

\section{End Points}

The primary end point of safety was evaluated by assessment of adverse events, clinical laboratory values, ECGs, vital signs, and physical examinations. ECG monitoring included standard 12-lead digital and 24-h ambulatory ECG (conducted at day 14 and week 16 visits); data were interpreted by a cardiologist at the Central ECG laboratory.

The primary efficacy end point was the absolute change in $\mathrm{FEV}_{1} \%$ predicted from baseline through week 16. Secondary efficacy end points were change from baseline through week 16 in sweat chloride concentration, change in weight, and patient-reported health using the disease-specific Cystic Fibrosis Questionnaire-Revised. ${ }^{15}$ Spirometry was performed according to American Thoracic Society guidelines. ${ }^{16}$ Sweat chloride collection was performed according to a standardized protocol using the Macroduct (Wescor, Inc) collection device ${ }^{17}$ and was evaluated centrally.

Analyses of other clinically relevant events, including time to first pulmonary exacerbation, duration and frequency of exacerbations using a modified Fuchs definition, ${ }^{18}$ hospitalizations, and use of antibiotics for sinopulmonary signs and symptoms, were prespecified tertiary end points. Investigator-reported pulmonary exacerbations were also captured as adverse events.

\section{Statistical Analyses}

Because the primary end point was safety, no formal sample size calculations were performed. Based on clinical considerations, a sample size of 120 subjects was selected to provide additional safety data on ivacaftor in this population. With 96 subjects in the ivacaftor group, the $90 \%$ CI estimates for the true adverse event rate were $7.4 \%$ and $19.5 \%$ if 12 subjects should have an adverse event, $2.8 \%$ and $12 \%$ should six subjects have an adverse event, and $0.4 \%$ and $6.4 \%$ should two subjects have an adverse event. The analysis suggests that this sample size was adequate to evaluate the safety of this drug in this population.

Analyses were performed on all randomized subjects who received at least one dose of the study drug. Analyses for safety are presented using descriptive statistics only and were not subjected to hypothesis testing. Primary analyses for efficacy end points were based on mixed-effects models for repeated measures with adjustments for age and baseline $\mathrm{FEV}_{1}$. Analyses of individual subject responses based on $\mathrm{FEV}_{1}$ and sweat chloride 
were performed using the predetermined criteria for enrollment in part B and two additional criteria, for a threshold response predetermined in the statistical analysis plan (ie, a mean decrease in sweat chloride of $\geq 10 \mathrm{mmol} / \mathrm{L}$ and $\geq 15 \mathrm{mmol} / \mathrm{L}$ from baseline), and differences between groups were compared using the Pearson $\chi^{2}$ test or Fisher exact test. Additional details on study methodology can be found in e-Appendix 2 .

\section{Results}

\section{Subjects}

The study was conducted between September 2009 and July 2010. Thirty-four study sites in the United States enrolled and randomized 140 subjects (ivacaftor, $\mathrm{n}=112$; placebo, $\mathrm{n}=28$ ). The groups were generally similar at baseline (Table 1 ).

A total of 104 subjects (92.9\%) in the ivacaftor group and 26 (92.9\%) in the placebo group completed dosing in part A (Fig 1). The most frequent reason for discontinuation was an adverse event (three subjects [2.7\%] in the ivacaftor group and two subjects [7.1\%] in the placebo group). Fortytwo ivacaftor subjects $(37.5 \%)$ and six placebo subjects $(21.4 \%)$ qualified for part B; the difference was not statistically significant $(P=.11)$.

\section{Safety Profile}

The overall proportion of subjects reporting any adverse event was similar between the groups, and the most common events were respiratory related (Table 2). Adverse events that occurred $\geq 5 \%$ more

Table 1-Baseline Characteristics of Study Participants

\begin{tabular}{|c|c|c|}
\hline Characteristic & Placebo $(n=28)$ & Ivacaftor $(\mathrm{n}=112)$ \\
\hline Female, No. (\%) & $12(43)$ & $54(48)$ \\
\hline \multicolumn{3}{|l|}{ Age, y } \\
\hline Mean (SD) & $25.0(8.4)$ & $22.8(10.3)$ \\
\hline Median & 24.0 & 19.5 \\
\hline Range & $12-39$ & $12-52$ \\
\hline \multicolumn{3}{|l|}{ Height, cm } \\
\hline Median & 170.7 & 164.0 \\
\hline Range & $145.6-184.0$ & 139.0-189.6 \\
\hline \multicolumn{3}{|l|}{ Weight, kg } \\
\hline Median & 64.9 & 55.9 \\
\hline Range & $44.2-100.3$ & $35.1-99.8$ \\
\hline \multicolumn{3}{|l|}{$\mathrm{BMI}, \mathrm{kg} / \mathrm{m}^{2}$} \\
\hline Median & 21.5 & 20.4 \\
\hline Range & $17.9-40.8$ & $15.7-31.6$ \\
\hline \multicolumn{3}{|c|}{ Sweat chloride, $\mathrm{mmol} / \mathrm{L}$} \\
\hline Mean (SD) & $102.4(7.9)$ & $101.4(10.3)$ \\
\hline Median & 101.5 & 101.0 \\
\hline Range & $91.0-122.0$ & $79.5-135.5$ \\
\hline \multicolumn{3}{|l|}{$\mathrm{FEV}_{1} \%$ predicted } \\
\hline Mean (SD) & $74.8(24.1)$ & $79.7(22.7)$ \\
\hline Median & 67.0 & 79.0 \\
\hline Range & $43-127$ & $40-129$ \\
\hline
\end{tabular}

None of the characteristics differed significantly between the groups ( $P>.05$ for all comparisons).

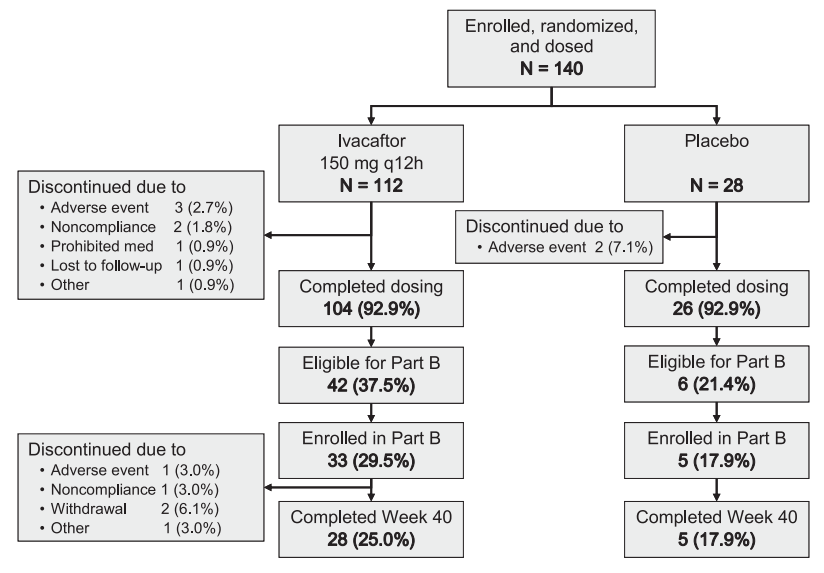

FIGURE 1. Subject flow diagram (following Consolidated Standards of Reporting Trials guidelines).

often in the ivacaftor group than in the placebo group were cough, nausea, rash, and contact dermatitis. None of these events was serious or led to study drug discontinuation. Conversely, investigator-identified pulmonary exacerbations occurred in a lower proportion of subjects treated with ivacaftor compared with placebo.

Adverse events were mostly mild $(35 \%)$ or moderate $(45 \%)$ in severity. One subject $(0.9 \%)$ in the ivacaftor group had three adverse events considered life threatening: fatigue, depression, and suicidal ideation. Ten subjects (8.9\%) in the ivacaftor group had 14 adverse events considered by the investigator to be severe; all were single events of nasal congestion, nasal polyps, epistaxis, increased viscosity of bronchial secretions, sinusitis, laryngitis, pulmonary exacerbation, diarrhea, dental caries, asthenia, rash, headache, arthritis, and nephrolithiasis. No severe events were reported in the placebo group. No deaths occurred during the study. Twenty-one subjects $(15.0 \%)$ had at least one event that met the criteria for a serious adverse event, including 15 (13.4\%) in the ivacaftor group and six $(21.4 \%)$ in the placebo group. This difference was predominantly due to pulmonary exacerbations, which were more frequent in the placebo group $(\mathrm{n}=5,17.9 \%)$ than in the ivacaftor group $(\mathrm{n}=10$, $8.9 \%)$. Other serious adverse events were single events of hemoptysis, hypoxia, nasal polyps, abdominal pain, myopathy, fatigue, depression, and suicidal ideation in the ivacaftor group; and bronchopneumonia, cognitive disorder, and venous thrombosis in the placebo group.

In the ivacaftor group, three subjects $(2.7 \%)$ were discontinued from the study because of a total of five events, which included asthenia, fatigue, and headache in one subject, arthritis in another, and myopathy in the third. In the placebo group, two subjects (7.1\%) were discontinued because of adverse events of abnormal feelings and cognitive disorder in one subject, 
and liver enzymes (alanine aminotransferase [ALT] and aspartate aminotransferase [AST]) elevated more than eight times the upper limit of normal (ULN) and increased lactate dehydrogenase levels in another subject. The liver enzyme abnormalities may have been due to concomitant use of an anabolic-steroidlike nutritional supplement. The liver enzyme elevations in the placebo subject were considered to be of moderate severity and resolved without treatment. The majority of subjects had ALT (90.7\%), AST (94.3\%), or total bilirubin $(98.6 \%)$ values that remained less than or equal to two times ULN. Four subjects (3.6\%) in the ivacaftor group and two $(7.1 \%)$ in the placebo group had at least one measured ALT and/or AST value greater than three times ULN. Study drug dosing was interrupted for two of these ivacaftor subjects, but alternative causes of liver enzyme increases were identified in both cases (gallstones with focal nodular hyperplasia and use of acetaminophen with alcohol); in both cases, dosing was resumed and tolerated well for the remainder of the treatment period. There were no clinically important trends attributable to ivacaftor identified in clinical laboratory (serum chemistry, hematology, coagulation studies, and urinalysis), vital sign, digital ECG, ambulatory ECG, or physical examination results.

\section{Efficacy Measures}

Lung Function Results: The adjusted mean absolute change from baseline through week 16 in $\mathrm{FEV}_{1} \%$ predicted in the ivacaftor group was an estimated treatment difference of $1.7 \%$ (95\% CI, -0.6 to 4.1 )

Table 2-Most Common Adverse Events Reported During the Study by Treatment Group

\begin{tabular}{lcc}
\hline \hline Adverse Event & $\begin{array}{c}\text { Placebo } \\
(\mathrm{n}=28)\end{array}$ & $\begin{array}{c}\text { Ivacaftor } \\
(\mathrm{n}=112)\end{array}$ \\
\hline Subjects with any adverse event & $25(89.3)$ & $98(87.5)$ \\
Cough & $4(14.3)$ & $34(30.4)$ \\
Pulmonary exacerbation ${ }^{\mathrm{a}}$ & $11(39.3)$ & $25(22.3)$ \\
Nasal congestion & $2(7.1)$ & $13(11.6)$ \\
Headache & $2(7.1)$ & $11(9.8)$ \\
Upper respiratory tract infection & $2(7.1)$ & $11(9.8)$ \\
Nausea & $1(3.6)$ & $10(8.9)$ \\
Oropharyngeal pain & $3(10.7)$ & $10(8.9)$ \\
Fatigue & $3(10.7)$ & $9(8.0)$ \\
Productive cough & $1(3.6)$ & $9(8.0)$ \\
Pyrexia & $2(7.1)$ & $9(8.0)$ \\
Rash & 0 & $9(8.0)$ \\
Sinusitis & $1(3.6)$ & $8(7.1)$ \\
Upper abdominal pain & $1(3.6)$ & $7(6.3)$ \\
Increase in C-reactive protein & $1(3.6)$ & $6(5.4)$ \\
Contact dermatitis & 0 & $6(5.4)$ \\
Diarrhea & $2(7.1)$ & $6(5.4)$ \\
\hline Data & &
\end{tabular}

Data are presented as No. (\%). Minimum frequency of $5 \%$ of subjects in the ivacaftor group.

${ }^{a}$ Coded as cystic fibrosis lung. compared with placebo; however, this difference was not statistically significant $(P=.15)$. This corresponded to a relative change of $2.4 \%$ (95\% CI, -0.9 to 5.8; $P=.16)$. No statistically significant differences were seen for other spirometric parameters (ie, FVC, forced expiratory flow at 25\%-75\% capacity). The number of subjects who qualified for the extension period on the basis of $\mathrm{FEV}_{1}$ improvement was also not statistically significant $(P=.46)$ between ivacaftor $(36[32.1 \%])$ and placebo (seven [25.0\%]).

Sweat Test Results: The adjusted mean sweat chloride concentration decreased slightly from baseline through week 16 in the ivacaftor group, whereas there was no change in placebo, with an estimated treatment difference of $-2.9 \mathrm{mmol} / \mathrm{L}$ (95\% CI, -5.6 to -0.2$)$; this difference was statistically significant $(P=.04)$. The change in sweat chloride occurred by day 15 and was sustained throughout the 16 weeks (Fig 2).

As described in the "Materials and Methods" section, (a priori) threshold changes in sweat chloride were established that would be used for analysis. A subset of ivacaftor subjects met one or more of these predetermined criteria for change in sweat chloride, whereas no placebo subjects met any of these criteria. Six subjects $(5.4 \%, P=.6)$ had a change in sweat chloride $\geq 15 \mathrm{mmol} / \mathrm{L}$ at both the day 15 and week 8 visits (the criterion for eligibility for part B), eight $(7.1 \%, P=.36)$ had a mean decrease of $\geq 15 \mathrm{mmol} / \mathrm{L}$ from baseline, and $17(15.2 \%, P=.02)$ had a mean decrease of $\geq 10 \mathrm{mmol} / \mathrm{L}$ from baseline averaged across all time points through week 16 .

Other Clinically Relevant Measures: There were no significant differences in either the ivacaftor or placebo group in the Cystic Fibrosis QuestionnaireRevised respiratory domain scores, pulmonary exacerbations, or antibiotic treatment of sinopulmonary signs or symptoms (Table 3). Change from baseline in weight and BMI (all subjects) and weight-for-age and BMI-for-age $z$ score (subjects $\leq 20$ years; ivacaftor, $\mathrm{n}=56$; placebo, $\mathrm{n}=7$ ) were not significantly different between arms.

\section{Open-Label Extension Period (Part B)}

Of 42 ivacaftor subjects in part A eligible for enrollment in part B, 33 entered the open-label period. Twenty-eight of these subjects met the $\mathrm{FEV}_{1}$ criterion and five met the sweat chloride criterion. Of six placebo subjects eligible for part $\mathrm{B}$, five subjects enrolled, all on the basis of $\mathrm{FEV}_{1}$ changes.

There were no meaningful findings regarding safety, lung function, and sweat chloride with continued treatment with ivacaftor. Further details of the results in part B are included in e-Appendix 2. 


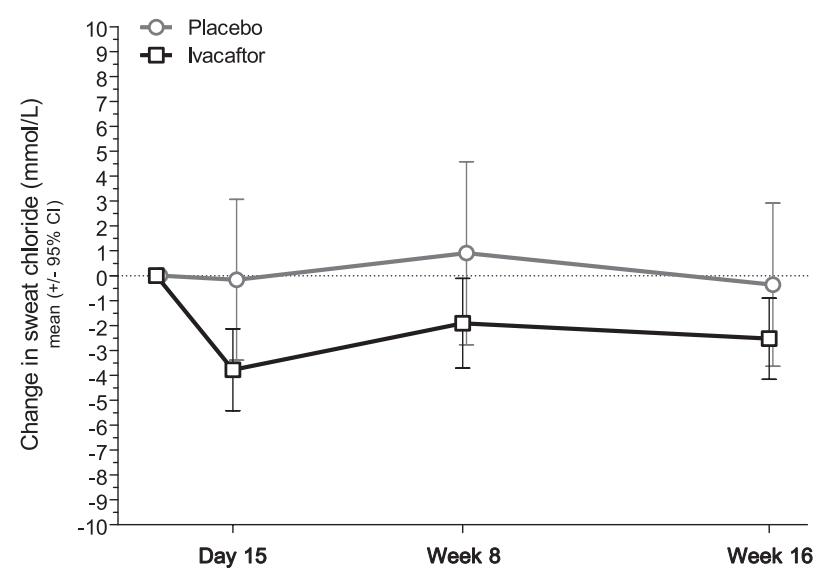

Figure 2. Average change from baseline ( $\pm 95 \%$ CI ) in sweat chloride concentration, a biomarker of cystic fibrosis transmembrane conductance regulator chloride transport activity. A greater proportion of subjects had a mean decrease from baseline through week 16 of $\geq 10 \mathrm{mmol} / \mathrm{L}$ (ivacaftor [15.2\%] vs placebo [0]; $P=.0240)$.

\section{DISCUSSION}

This randomized, double-blind, placebo-controlled, multicenter study evaluated the safety and efficacy of the CFTR potentiator, ivacaftor, in subjects with CF homozygous for the F508del-CFTR mutation. The safety profile of ivacaftor compared with placebo in this study supports further clinical evaluation in patients with CF. Treatment with ivacaftor was not associated with significant improvements in $\mathrm{FEV}_{1}$ or other clinical end points. There was a small reduction in sweat chloride concentration observed during the randomized controlled portion of the study that was not reflected in the population enrolled in the openlabel extension.

Gaining additional safety experience with ivacaftor was a primary study objective. The incidence of adverse events was generally similar between ivacaftor and placebo, with many of the most common events being respiratory related, as expected in patients with CF. Cough, rash, and contact dermatitis occurred more frequently in the ivacaftor group, whereas pulmonary exacerbation as defined by the treating physician was reported more frequently in the placebo group. The numeric differences between arms may be due to the small sample size in the placebo group resulting from the skewed randomization. All adverse events considered severe by the investigator were reported in the ivacaftor group. One possible explanation for this finding is chance, given that more subjects were randomized to ivacaftor than to placebo (4:1 ratio); there would need to be only two subjects (about 7\%) in the placebo group for there to be no difference. Conversely, there was a higher frequency of serious adverse events (eg, hospitalization) reported in the placebo group, supporting the conclusion that treatment with ivacaftor does not lead to clinically important safety concerns. No new safety events arose during the 40-week open-label extension.

Another objective of the study was to explore potential clinical improvement with ivacaftor treatment, although the study was powered based on safety, not efficacy. There were no statistically significant improvements in pulmonary function with ivacaftor treatment in F508del-CFTR homozygous subjects. Similarly, no notable changes were observed for other clinical end points, including weight gain and patientreported respiratory symptoms. A numeric difference between treatment groups in the risk of pulmonary exacerbations over 16 weeks did not achieve statistical significance. It should be noted that some subjects in the placebo group met the criterion for Part B by experiencing an improvement in their $\mathrm{FEV}_{1}$. Such an increase in some subjects is not unusual in CF trials. Possible explanations include artificially low baseline and regression to mean, a change related to chronic therapies (eg, inhaled cycled antibiotics), or improvement due to antibiotic use during exacerbation.

Sweat chloride concentration, a measure of CFTR function, showed a small decrease $(2.9 \mathrm{mmol} / \mathrm{L}) \mathrm{com}$ pared with placebo through 16 weeks of therapy, although this change was not sustained for the majority of subjects in the open-label extension period. By contrast, ivacaftor demonstrated a median change

Table 3-Other Clinically Relevant Measures

\begin{tabular}{|c|c|c|}
\hline Measure & Placebo $(\mathrm{n}=28)$ & Ivacaftor $(\mathrm{n}=112)$ \\
\hline Absolute change in $\mathrm{FEV}_{1} \%$ predicted & -0.2 & 1.5 \\
\hline Absolute change in CFQ- $\mathrm{R}$ respiratory domain score & -1.4 & -0.1 \\
\hline Absolute change in weight, $\mathrm{kg}$ & 0.96 & 0.84 \\
\hline Absolute change in BMI, kg/m² & 0.25 & 0.21 \\
\hline Absolute change in weight-for-age $z$ score & 0.0007 & 0.43 \\
\hline Absolute change in BMI-for-age $z$ score & -0.002 & 0.73 \\
\hline Pulmonary exacerbation, ${ }^{\mathrm{a}}$ No. $(\%)$ & $10(32)$ & $25(21)$ \\
\hline Antibiotic treatment of sinopulmonary signs or symptoms, ${ }^{b}$ No. (\%) & $16(56)$ & $48(43)$ \\
\hline
\end{tabular}

$P$ values for all measures were not significant. CFQ-R $=$ Cystic Fibrosis Questionnaire-Revised.

aTreatment with new or changed antibiotic therapy for $\geq 4$ sinopulmonary signs/symptoms.

bEvents requiring new or changed antibiotic therapy for $\geq 1$ sinopulmonary sign/symptom. 
of $-59.5 \mathrm{mmol} / \mathrm{L}$ after 28 days at the same dose in the G551D-CFTR population. ${ }^{12}$ Moreover, the G551D-CFTR trial also showed a significant improvement in $\mathrm{FEV}_{1}$. Interestingly, in the current study of F508del-CFTR homozygotes, $15.2 \%$ of ivacaftor subjects demonstrated an average sweat chloride reduction of $\geq 10 \mathrm{mmol} / \mathrm{L}$ across all time points through week 16, compared with no subjects in the placebo group. This may indicate a subpopulation of subjects homozygous for the F508del-CFTR mutation with some F508del-CFTR protein that reaches the epithelial cell surface, supporting in vitro evidence. One in vitro study tested ivacaftor in human bronchial epithelial (HBE) cells from subjects with CF who were homozygous for the F508del-CFTR mutation. ${ }^{10}$ Among $\mathrm{HBE}$ that showed potentiation by ivacaftor, the effect size (up to $16 \%$ of normal activity) was considerably lower than in HBE from a G551D/F508del donor (nearly $50 \%$ normal).

\section{ConClusions}

This study augments the safety information for ivacaftor and supports the continued evaluation of ivacaftor in subjects with CF. The lack of an observed clinical effect suggests that CFTR potentiation with ivacaftor alone will not benefit patients with $\mathrm{CF}$ who are homozygous for the F508del-CFTR mutation. Although a proportion of subjects treated with ivacaftor showed a small change in the sweat chloride biomarker, this was not associated with clinical benefit. Additional studies are needed to explore any relationship between sweat chloride and a clinical response in this group. Alternative therapeutic approaches, possibly using a corrector compound to increase the amount of F508del-CFTR protein that reaches the epithelial cell surface, should be evaluated for clinical benefit in this population.

\section{ACKNOWLEDGMENTS}

Author contributions: Dr Flume served as guarantor of the manuscript.

Dr Flume: contributed to the acquisition of data, analysis and interpretation of data, drafting of the manuscript, critical revision of the manuscript for important intellectual content, and approval of the final version.

Dr Liou: contributed to the acquisition of data and critical revision of the manuscript for important intellectual content and saw and approved the final version.

Dr Borowitz: contributed to the acquisition of data and critical revision of the manuscript for important intellectual content and saw and approved the final version.

Dr Li: contributed to the analysis and interpretation of the data, statistical analysis and critical revision of the manuscript for important intellectual content and saw and approved the final version. Dr Yen: contributed to the concept and design of the study, analysis and interpretation of data, and critical revision of the manuscript for important intellectual content and saw and approved the final version.
Dr Ordoñez: contributed to the concept and design of the study, analysis and interpretation of data, and critical revision of the manuscript for important intellectual content and saw and approved the final version.

Dr Geller: contributed to the acquisition of data and critical revision of the manuscript for important intellectual content and saw and approved the final version.

Financial/nonfinancial disclosures: The authors have reported to CHEST the following conflicts of interest: Dr Flume has received grants from the Cystic Fibrosis Foundation and received funding to participate in this clinical trial. Dr Liou has received funding from the National Institutes of Health/National Heart, Lung and Blood Institute, the Cystic Fibrosis Foundation, and the Ben B. and Ira M. Margolis Family Foundation of Utah for work related to CF. He is a member of the CHEST Editorial Board and Cystic Fibrosis Foundation Patient Registry Data Use Committee. He is a steering committee consultant on the Registry to Evaluate Early and Long-term Pulmonary Arterial Hypertension Disease Management (REVEAL) project on pulmonary arterial hypertension sponsored by Actelion Pharmaceuticals Ltd and was formerly a steering committee member on the Epidemiologic Study of Cystic Fibrosis project on CF sponsored by Genentech, Inc. As the principal investigator for the Therapeutic Development Network Center at the University of Utah, Dr Liou has received funding to participate in trials of novel treatments for CF from Altus Pharmaceuticals Inc; AXCAN Scandipharm Inc; Bayer; Boehringer Ingelheim GmbH; Genentech, Inc; Gilead; Inspire; KaloBios Pharmaceuticals, Inc; MPEX Pharmaceuticals, Inc; and Vertex Pharmaceuticals Incorporated. Dr Liou has consulted for the Gehrson Lehman Group, Inc and has provided expert legal testimony. Dr Borowitz was a member of the Vertex Global Advisory Committee and has received funding to participate in Vertex Pharmaceuticals Incorporated clinical trials. Drs Li, Yen, and Ordoñez are employees and stockholders of Vertex Pharmaceuticals Incorporated. Dr Geller has consulted for CSL Behring, Aradigm Corporation, Novartis AG, Gilead, and Genentech, Inc. He has received funding to participate in trials of novel treatments for CF from Novartis AG, Gilead, MPEX Pharmaceuticals, Inc, Bayer, INSMED Incorporated, Pharmaxis Ltd, Inspire, and Boehringer Ingelheim GmbH.

Role of sponsors: Vertex Pharmaceuticals Incorporated provided the financial support and study medication for the conduct of the study. The Cystic Fibrosis Foundation had no role in the design of the study, the collection and analysis of the data, or in the preparation of the manuscript.

Other contributions: The authors thank all the patients and families who participated in this research and the nurses and technicians who made this research possible. The authors also thank the following employees of Vertex Pharmaceuticals Incorporated: Nikki Shannon, RN, BHScA, for clinical operations support; Tatyana Wanderer, PhD, for study document preparation; and Adrienne Aiello, PharmD, and Barry Lubarsky, PhD, for coordinating the editing of the submitted manuscript.

Additional information: The e-Appendixes can be found in the "Supplemental Materials" area of the online article.

\section{REFERENCES}

1. Rowe SM, Miller S, Sorscher EJ. Cystic fibrosis. $N$ Engl J Med. 2005;352(19):1992-2001.

2. McKone EF, Emerson SS, Edwards KL, Aitken ML. Effect of genotype on phenotype and mortality in cystic fibrosis: a retrospective cohort study. Lancet. 2003;361(9370):1671-1676.

3. Kerem B, Rommens JM, Buchanan JA, et al. Identification of the cystic fibrosis gene: genetic analysis. Science. 1989; 245(4922):1073-1080.

4. Riordan JR, Rommens JM, Kerem B, et al. Identification of the cystic fibrosis gene: cloning and characterization of complementary DNA. Science. 1989;245(4922):1066-1073.

5. Rommens JM, Iannuzzi MC, Kerem B, et al. Identification of the cystic fibrosis gene: chromosome walking and jumping. Science. 1989;245(4922):1059-1065. 
6. Cystic Fibrosis Foundation Patient Registry. 2009 Annual Data Report to the Center Directors. Bethesda, MD: 2010.

7. Denning GM, Anderson MP, Amara JF, Marshall J, Smith AE, Welsh MJ. Processing of mutant cystic fibrosis transmembrane conductance regulator is temperature-sensitive. Nature. 1992;358(6389):761-764.

8. Kartner N, Augustinas O, Jensen TJ, Naismith AL, Riordan JR. Mislocalization of delta F508 CFTR in cystic fibrosis sweat gland. Nat Genet. 1992;1(5):321-327.

9. Van Goor F, Straley KS, Cao D, et al. Rescue of DeltaF508CFTR trafficking and gating in human cystic fibrosis airway primary cultures by small molecules. Am J Physiol Lung Cell Mol Physiol. 2006;290(6):L1117-L1130.

10. Van Goor F, Hadida S, Grootenhuis PD, et al. Rescue of $\mathrm{CF}$ airway epithelial cell function in vitro by a CFTR potentiator, VX-770. Proc Natl Acad Sci U S A. 2009;106(44): 18825-18830.

11. Bobadilla JL, Macek M Jr, Fine JP, Farrell PM. Cystic fibrosis: a worldwide analysis of CFTR mutations-correlation with incidence data and application to screening. Hum Mutat. 2002;19(6):575-606.

12. Accurso FJ, Rowe SM, Clancy JP, et al. Effect of VX-770 in persons with cystic fibrosis and the G551D-CFTR mutation. N Engl J Med. 2010;363(21):1991-2003.
13. Bronsveld I, Mekus F, Bijman J, et al. Chloride conductance and genetic background modulate the cystic fibrosis phenotype of Delta F508 homozygous twins and siblings. J Clin Invest. 2001;108(11):1705-1715.

14. Veeze HJ, Halley DJ, Bijman J, de Jongste JC, de Jonge HR, Sinaasappel M. Determinants of mild clinical symptoms in cystic fibrosis patients. Residual chloride secretion measured in rectal biopsies in relation to the genotype. J Clin Invest. 1994;93(2):461-466.

15. Quittner AL, Buu A, Messer MA, Modi AC, Watrous M. Development and validation of The Cystic Fibrosis Questionnaire in the United States: a health-related quality-of-life measure for cystic fibrosis. Chest. 2005;128(4):2347-2354.

16. American Thoracic Society. Standardization of spirometry, 1994 update. Am J Respir Crit Care Med. 1995;152(3): 1107-1136.

17. Rose JB, Ellis L, John B, et al. Does the Macroduct collection system reliably define sweat chloride concentration in subjects with intermediate results? Clin Biochem. 2009;42(12):1260-1264.

18. Fuchs HJ, Borowitz DS, Christiansen DH, et al; The Pulmozyme Study Group. Effect of aerosolized recombinant human DNase on exacerbations of respiratory symptoms and on pulmonary function in patients with cystic fibrosis. $N \mathrm{Engl}$ J Med. 1994;331(10):637-642. 